\title{
Editorial The dentist's expanding role in orofacial pain
}

Pain is one of the most powerful negative emotions humans experience. It demands attention and response. Acute pain alarms the individual to the injury allowing the sufferer to address the threat. Acute pain provides protection from environment challenges. It is basic to survival and therefore has purpose. However, some pains last far longer than normal healing time and therefore no longer have protective value. These pains are termed chronic. These pains can become destructive to the human spirit, leading to significant reduction in the quality of life. Some of the most common types of chronic pains originate from musculoskeletal structures. Chronic back and neck pain are very frequent in the general population. The 12 -month prevalence of neck pain ranges between $30 \%$ and $50 \%$ [1]. In one study [2], 33\% of 3064-year-old workers reported having musculoskeletal pain within the last month and $20 \%$ of them reported multiple sites of pain. These pains are typically dull achy pains that can significantly decrease the individual's ability to function. Musculoskeletal pains that originate in the masticatory structures are known as temporomandibular disorders (TMDs) [3]. It is estimated that $10 \%$ of the general population over the age of 18 has TMD pain [4]. The prevalence of orofacial pain in the general population at age 30-31 years has been reported to be $23 \%$ [5]. In another study [6], $23-24 \%$ of 45 -year-olds in the general population report pain during chewing.

For many years the dental profession has appreciated that TMD may be related to occlusal factors. We have also come to appreciate that not all TMDs are related to the occlusion and certainly not all orofacial pains are related to TMD [7]. Therefore, a basic obligation we have to our patients is in determining those pain conditions that relate to the occlusion from those that do not. For if a TMD is related to the occlusion, dentists must stand up and manage the condition, since we are the only health care providers trained to treat occlusion. On the other hand, if the patient is suffering from a TMD or another orofacial pain that is unrelated to the occlusion, it would be wrong to provide occlusal therapy since this would prove to be ineffective. Therefore, there is a great burden on the dental profession to determine the role of the occlusion in the patient's pain condition before permanent treatment is begun. This may not be an easy task but it is basic to helping our patients while doing no harm to them.

Correspondence: Jeffrey P. Okeson, DMD, D-142, Orofacial Pain Center, University of Kentucky College of Dentistry, Lexington, KY 40536-0297, USA. E-mail: okeson@uky.edu
The problem we face is that exposure to TMD in many dental schools is minimal and therefore most clinicians learn about these patients when they present for treatment in the dental practice. Unfortunately as the dentist peruses more knowledge regarding these problems the learning that may occur in uncontrolled settings and without scientific evidence. This leads to differing philosophies and confusion for the clinician and often poor, misdirected treatment for the patient. Dental schools and the dental profession need to offer better, scientifically based, educational programs so that clinicians can more successfully diagnose and manage their patients.

To further challenge the profession is the reality that the field of orofacial pain is very complicated and multifaceted [8]. It stretches beyond that of traditional dentistry. Therefore, the dental profession will need to move outside of previously established concepts and expand into new arenas of knowledge. This may prove difficult as we move out of our traditional comfort zones. We may be required to give up strongly held beliefs to the evidence-based science of pain.

Helping patients with chronic orofacial pain begins with understanding the precise condition of which the patients are suffering. Not all pains are the same and therefore treatments are different. The most critical task the clinician can do for the orofacial pain patient is to establish the correct diagnosis. This is basic to select the most appropriate treatment. In fact, the most common reason for treatment failure is not the quality of the treatment the patent had received. It is a misdiagnosis. Often we find patients who have received excellent dental treatments but these treatments did not resolve the pain complaint because they were not directed to the etiology of the problem. In order to be successful, the clinician must have a thorough appreciation for the types of orofacial pain disorders that present in a dental practice so a proper diagnosis can be established.

The dental profession is facing a significant challenge. There are many patients in the general population who are suffering from orofacial pain. We need to possess the knowledge to take adequately care for these individuals. Yet in the past TMD and other orofacial pain disorders have not been taught well in our dental education systems. This creates a significant professional problem. How can a clinician identify a disorder that he or she has not heard about? Yet this is a critical issue since making the proper diagnosis is basic to select the appropriate treatment. This is also the precise reason why we need journals like this to help provide better evidence-based information for practitioners so they can better care for their patients. Our profession was established to help reduce the suffering of 
our fellow men and women. We need to provide the education necessary to accomplish this goal. Education is where this begins. It is my hope that we can continue to expand our knowledge in the field of orofacial pain so that we can better reduce the suffering of our patients. This is the basic mission of our profession.

Jeffrey P. Okeson E-mail: okeson@uky.edu

\section{Conflict of interest}

The authors declare that there is no conflict of interest.

\section{References}

[1] Hogg-Johnson S, van der Velde G, Carroll LJ, Holm LW, Cassidy JD, Guzman J, et al. The burden and determinants of neck pain in the general population: results of the Bone and Joint Decade 2000-2010
Task Force on Neck Pain and Its Associated Disorders. Spine (Phila Pa 1976) 2008;33(4 Suppl.):S39-51.

[2] Miranda H, Kaila-Kangas L, Heliovaara M, Leino-Arjas P, Haukka E, Liira J, et al. Musculoskeletal pain at multiple sites and its effects on work ability in a general working population. Occup Environ Med 2009.

[3] De Leeuw R. Orofacial pain: guidelines for classification, assessment, and management, 4th edn. Chicago: Quintessence Publ. Co.; 2008.

[4] LeResche L. Epidemiology of temporomandibular disorders: implications for the investigation of etiologic factors. Crit Rev Oral Biol Med 1997;8(3):291-305.

[5] Macfarlane TV, Kenealy P, Kingdon HA, Mohlin B, Pilley JR, Mwangi $\mathrm{CW}$, et al. Orofacial pain in young adults and associated childhood and adulthood factors: results of the population study, Wales, United Kingdom. Community Dent Oral Epidemiol 2009;37(5):438-50.

[6] Riley JL 3rd, Gilbert GH. Orofacial pain symptoms: an interaction between age and sex. Pain 2001;90(3):245-56.

[7] Okeson JP. Management of temporomandibular disorders and occlusion, 6th edn. St Louis, MO: Mosby Year Book Publ; 2008.

[8] Okeson JP. Bell's Orofacial pains, 6th edn. Chicago, IL: Quintessence Publishing Co. Inc.; 2005. 